Supporting Information

\title{
Quantitative Measurement of Li-Ion Concentration and Diffusivity in Solid-State Electrolyte
}

Gun Park', Hongjun Kimt', Jimin Oht, \#, Youngwoo Choit, Olga S. Ovchinnikovas, Seokhwan Mint, Young-Gi Lee", and Seungbum Hongt,*

†Department of Materials Science and Engineering, Korea Advanced Institute of

Science and Technology, 291 Daehak-ro, Guseong-dong, Yuseong-gu, Daejeon

34141, Republic of Korea

\#ICT Creative Research Laboratory, Electronics and Telecommunications Research

Institute, 218 Gajeong-ro, Yuseong-gu, Daejeon 34129, Republic of Korea

$\S$ Center for Nanophase Materials Sciences, Oak Ridge National Laboratory, Oak

Ridge, Tennessee 37831, United States 
*Corresponding Author: seungbum@kaist.ac.kr

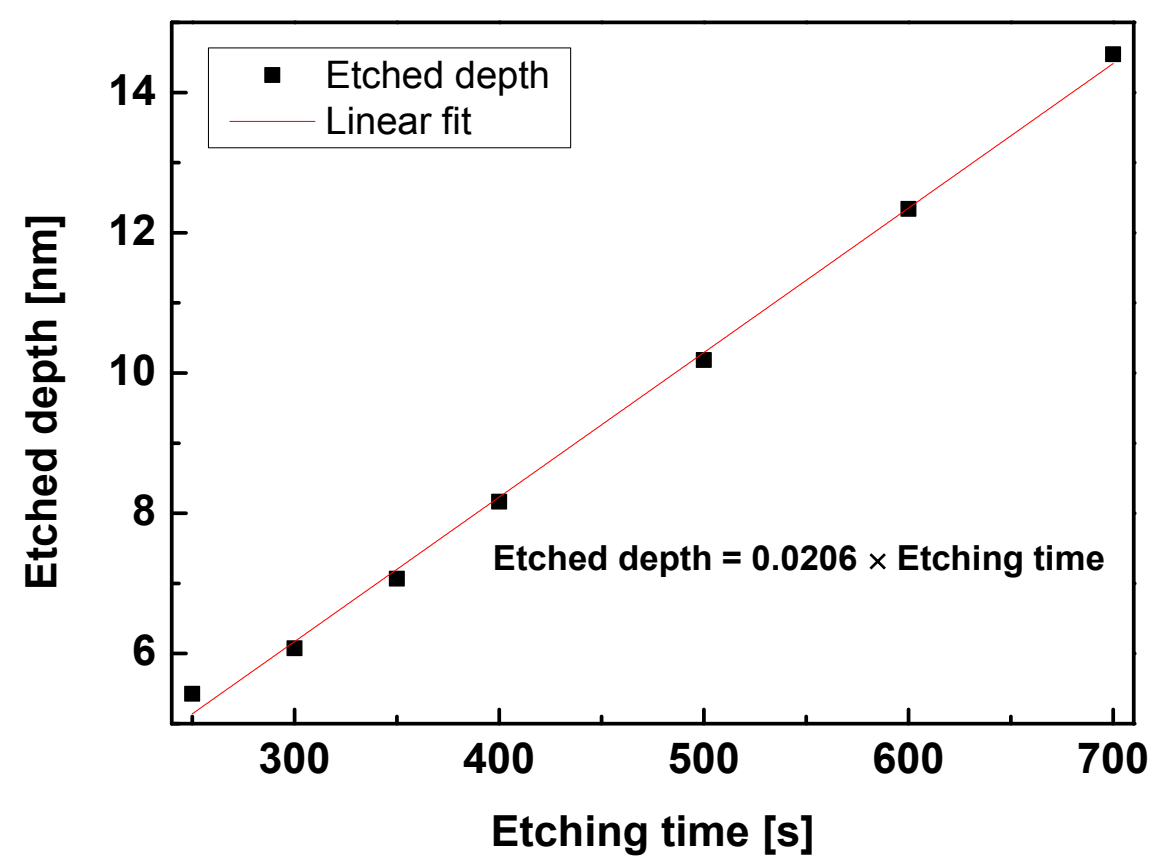

Figure S1. Plot of depth of trenches as a function of etching time (black) measured by 3D optical microscope and linear fitting result (red) with corresponding linear function.

Due to the limitation of spatial resolution of 3D optical microscope, we measured the depth of trenches sufficiently etched for 250 700 s and extrapolated the depth using the linear function as shown in Figure S1. 


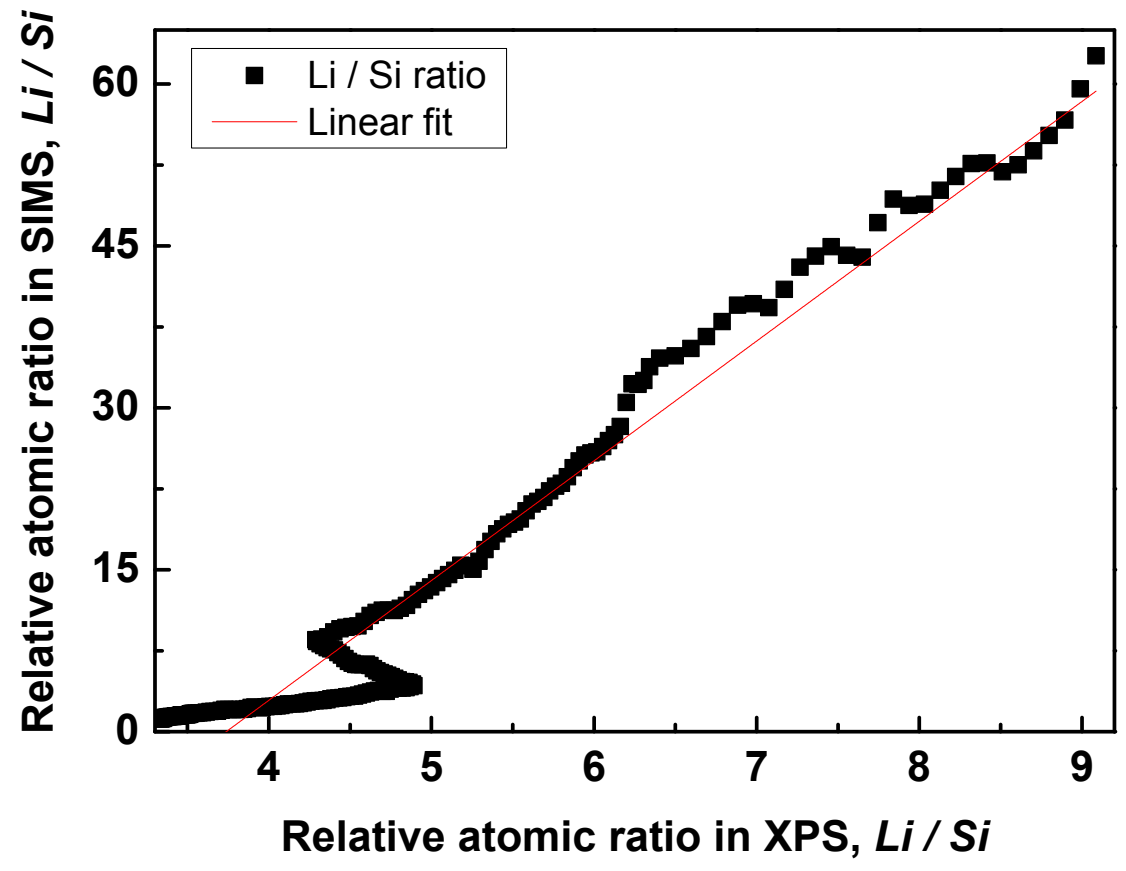

Figure S2. Plot of relative atomic ratio between $\mathrm{Li}$ and Si based on the SIMS and XPS depth profiling results (black) and corresponding linear fitting result (red).

We compared the relative atomic ratio between $\mathrm{Li}$ and $\mathrm{Si}\left(L i / S_{I}\right)$ assuming that the amount of $\mathrm{Si}$ is constant as a function of depth to verify SIMS and XPS are strongly correlated with each other. We obtained high R-square value (0.945) based on the linear fitting as shown in Figure S2. 
a

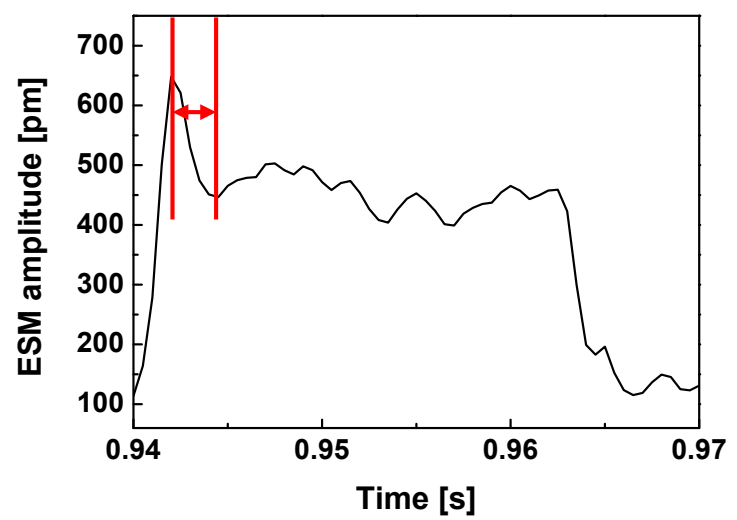

b

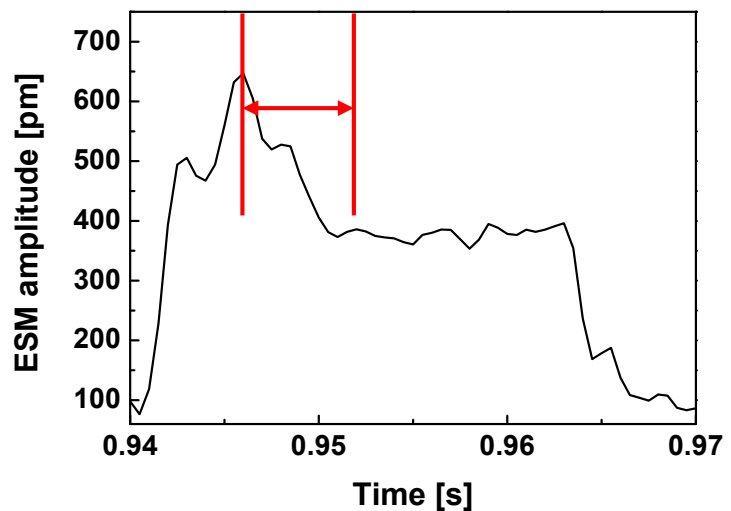

Figure S3. The ESM amplitude showing premature relaxation behavior of Li-ion before turning the DC bias voltage off (red region in Figure S3a and b). Figure S3 was obtained under the same experimental condition as we used in Figure 3. 


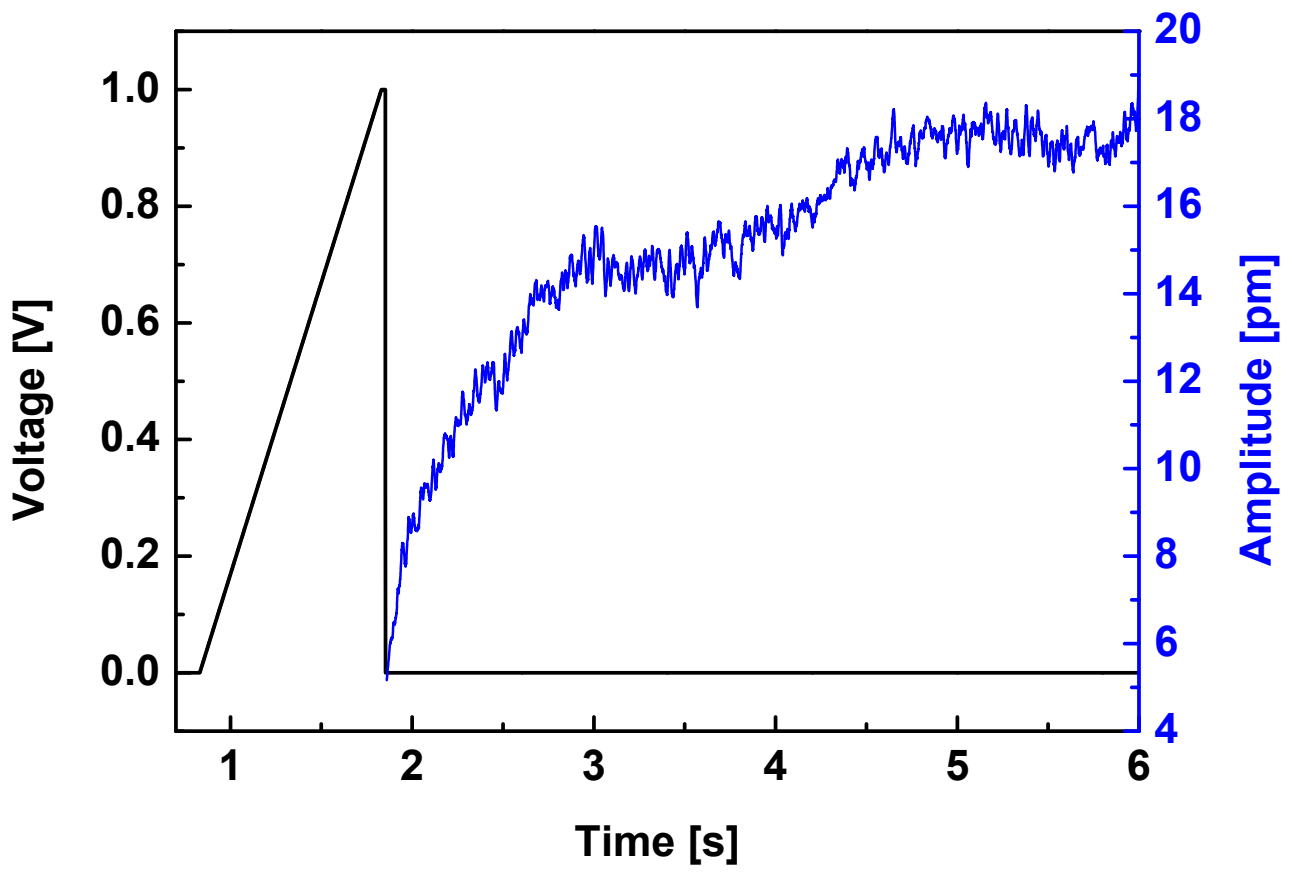

Figure S4. The ESM relaxation curve after applying a positive triangular form of DC voltage pulse.

The lower amplitude increased immediately after removal of positive DC voltage, which assures that this amplitude is a signal from ionic movement. Depending on the polarity of DC bias, the trend of amplitude change was reversed. 


\begin{tabular}{c|c|c}
\hline Element & $\begin{array}{c}\text { Wavelength } \\
(\mathrm{nm})\end{array}$ & $\begin{array}{c}\text { Calculated concentration } \\
(\mathrm{mg} / \mathrm{kg})\end{array}$ \\
\hline $\mathrm{Al}$ & 396.152 & 40626 \\
\hline $\mathrm{Ge}$ & 209.426 & 61139 \\
\hline $\mathrm{Li}$ & 670.783 & 19709 \\
\hline $\mathrm{P}$ & 213.618 & 157249 \\
\hline $\mathrm{Ti}$ & 336.122 & 26725 \\
\hline
\end{tabular}

Table S1. The ICP-OES result showing the analyze wavelength and calculated concentration for each element. 
